\title{
A Subclass of Harmonic Functions Associated with Wright's Hypergeometric Functions
}

\author{
Gangadharan Murugusundaramoorthy, Kalliyapan Vijaya \\ School of Advanced Sciences, Vellore Institute of Technology University, Vellore, India \\ E-mail:gmsmoorthy@yahoo.com,kvavit@yahoo.co.in \\ Received May 7, 2010; revised May 21, 2010; accepted June 25, 2010
}

\begin{abstract}
We introduce a new class of complex valued harmonic functions associated with Wright hypergeometric functions which are orientation preserving and univalent in the open unit disc. Further we define, Wright generalized operator on harmonic function and investigate the coefficient bounds, distortion inequalities and extreme points for this generalized class of functions.
\end{abstract}

Keywords: Harmonic Univalent Starlike Functions, Harmonic Convex Functions, Wright Hypergeometric Functions, Raina-Dziok Operator, Distortion Bounds, Extreme Points

\section{Introduction}

A continuous function $f=u+i v$ is a complex-valued harmonic function in a complex domain $G$ if both $u$ and $v$ are real and harmonic in $G$. In any simply-connected domain $D \subset$ G, we can write $f=h+\bar{g}$, where $h$ and $g$ are analytic in $D$. We call $h$ the analytic part and $g$ the co-analytic part of $f$. A necessary and sufficient condition for $f$ to be locally univalent and orientation preserving in $D$ is that $\left|h^{\prime}(z)\right|>\left|g^{\prime}(z)\right|$ in $D$ (see [1]).

Denote by $\mathrm{H}$ the family of functions

$$
f=h+\bar{g}
$$

which are harmonic, univalent and orientation preserving in the open unit disc $U=\{z:|z|<1\}$ so that $\mathrm{f}$ is normalized by $f(0)=h(0)=f_{z}(0)-1=0$. Thus, for $f=h+\bar{g}$ $\in \mathrm{H}$, we may express

$$
f(z)=z+\sum_{m=2}^{\infty} a_{m} z^{m}+\overline{\sum_{m=1}^{\infty} b_{m} z^{m}},\left|b_{1}\right|<1,
$$

where the analytic functions $h$ and $g$ are in the forms

$$
h(z)=z+\sum_{m=2}^{\infty} a_{m} z^{m}, g(z)=\sum_{m=1}^{\infty} b_{m} z^{m} \quad\left(\left|b_{1}\right|<1\right) .
$$

We note that the family $H$ of orientation preserving, normalized harmonic univalent functions reduces to the well known class $S$ of normalized univalent functions if the co-analytic part of $f$ is identically zero, that is $g \equiv 0$.

Let the Hadamard product (or convolution) of two power series

$$
\phi(z)=z+\sum_{m=2}^{\infty} \phi_{m} z^{m}
$$

and

$$
\psi(z)=\sum_{m=2}^{\infty} \psi_{m} z^{m}
$$

in $S$ be defined by

$$
\left(\phi^{*} \psi\right)(z)=\sum_{m=2}^{\infty} \phi_{m} \psi_{m} z^{m}
$$

For positive real parameters $\alpha_{1}, \mathrm{~A}_{1}, \ldots, \alpha_{p}, A_{p}$ and $\beta_{1}, \mathrm{~B}_{1}, \ldots, \beta_{q} B_{q}(p, q \in N=1,2,3, \ldots)$ such that

$$
1+\sum_{m=1}^{q} B_{m},-\sum_{m=1}^{p} A_{m} \geq 0, \quad z \in U .
$$

The Wright's generalized hypergeometric function [2]

$$
\begin{aligned}
& p \Psi_{q}\left[\left(\alpha_{1}, A_{1}\right), \cdots,\left(\alpha_{p}, A_{p}\right) ;\left(\beta_{1}, B_{1}\right) \cdots\left(\beta_{q}, B_{q}\right) ; z\right] \\
& =p \Psi_{q}\left[\left(\alpha_{m}, A_{m}\right)_{1, p}\left(\beta_{m}, B_{m}\right)_{1, q} ; z\right]
\end{aligned}
$$

is defined by

$$
\begin{aligned}
& \Psi_{q}\left[\left(\alpha_{t}, A_{t}\right)_{1, p}\left(\beta_{t}, B_{t}\right)_{1, q} ; z\right] \\
= & \sum_{m=0}^{\infty}\left\{\prod_{t=0}^{p} \Gamma\left(\alpha_{t}+m A_{t}\right)\right\}\left\{\prod_{t=0}^{q} \Gamma\left(\beta_{t}+m B_{t}\right)\right\}^{-1} . \\
& \frac{z^{m}}{m !}, \quad z \in U .
\end{aligned}
$$

If $A_{t}=1(t=1,2, p)$ and $B_{t}=1(t=1,2, q)$ we have 
the relationship:

$$
\begin{aligned}
& \Omega_{p} \Psi_{q}\left[\left(\alpha_{t}, A_{t}\right)_{1, p}\left(\beta_{t}, B_{t}\right)_{1, q} ; z\right] \\
& ={ }_{p} F_{q}\left(\alpha_{1}, \cdots \alpha_{p} ; \beta_{1} \cdots, \beta_{q} ; z\right) \\
& =\sum_{m=0}^{\infty} \frac{\left(\alpha_{1}\right)_{m}, \cdots\left(\alpha_{p}\right)_{m}}{\left(\beta_{1}\right)_{m} \cdots,\left(\beta_{q}\right)_{m}} \frac{z^{m}}{m !},
\end{aligned}
$$

$\left(p \leq q+1 ; p . q \in N_{0}=N \cup\{0\} ; z \in U\right)$ is the generalized hypergeometric function (see for details [3]) where $N$ denotes the set of all positive integers and $(\alpha)_{n}$ is the Pochhammer symbol and

$$
\Omega=\left\{\prod_{t=0}^{p} \Gamma\left(\alpha_{t}\right)\right\}^{-1}\left\{\prod_{t=0}^{q} \Gamma\left(\beta_{t}\right)\right\}
$$

By using the generalized hypergeometric function Dziok and Srivastava [3] introduced the linear operator. In [4] Dziok and Raina extended the linear operator by using Wright generalized hypergeometric function. First we define a function

$$
\begin{aligned}
& \Phi_{q}\left[\left(\alpha_{t}, A_{t}\right)_{1, p}\left(\beta_{t}, B_{t}\right)_{1, q} ; z\right] \\
= & \Omega_{p} \Psi_{q}\left[\left(\alpha_{t}, A_{t}\right)_{1, p}\left(\beta_{t}, B_{t}\right)_{1, q} ; z\right]
\end{aligned}
$$

Let $W\left[\left(\alpha_{t}, A_{t}\right)_{1, p}\left(\beta_{t}, B_{t}\right)_{1, q}\right]: S \rightarrow S$ be a linear operator defined by

$$
\begin{gathered}
W\left[\left(\alpha_{t}, A_{t}\right)_{1, p}\left(\beta_{t}, B_{t}\right)_{1, q}\right] \varphi(z) \\
:=z_{p} \Phi_{q}\left[\left(\alpha_{t}, A_{t}\right)_{1, p}\left(\beta_{t}, B_{t}\right)_{1, q} ; z\right] * \varphi(z)
\end{gathered}
$$

We observe that, for $f(z)$ of the form (1), we have

$$
\begin{aligned}
& W\left[\left(\alpha_{t}, A_{t}\right)_{1, p}\left(\beta_{t}, B_{t}\right)_{1, q}\right] \varphi(z) \\
:= & z+\sum_{m=2}^{\infty} \sigma_{m}\left(\alpha_{1}\right) \varphi_{m} z^{n},
\end{aligned}
$$

where $\sigma_{m}\left(\alpha_{1}\right)$ is defined by

$$
\begin{aligned}
\sigma_{m}\left(\alpha_{1}\right) & \frac{\Omega \Gamma\left(\alpha_{1}+A_{1}(m-1)\right) \cdots \Gamma\left(\alpha_{p}+A_{p}(m-1)\right)}{(m-1) ! \Gamma\left(\beta_{1}+B_{1}(m-1)\right) \cdots \Gamma\left(\beta_{q}+B_{q}(m-1)\right)}
\end{aligned}
$$

If, for convenience, we write

$$
\begin{aligned}
& W\left[\alpha_{1}\right] \phi(z) \\
= & W\left[\left(\alpha_{1}, A_{1}\right), \cdots\left(\alpha_{p}, A_{p}\right) ;\left(\beta_{1}, B_{1}\right) \cdots\left(\beta_{q}, B_{q}\right)\right] \phi(z)
\end{aligned}
$$

introduced by Dziok and Raina [4].

It is of interest to note that, if $A_{t}=1(t=1,2, \ldots, p), B_{t}$ $=1(t=1,2, \ldots, q)$ in view of the relationship (6) the linear operator (8) includes the Dziok-Srivastava operator (see [3]), for more details on these operators see $[3,4,6,7]$ and
[8]. It is interesting to note that Wright generalized hypergeometric function contains, Dziok-Srivastava operator as its special cases, further other linear operators the Hohlov operator, the Carlson-Shaffer operator [6], the Ruscheweyh derivative operator [7], the generalized Bernardi-Libera-Livingston operator, the fractional derivative operator [8], and so on. For example if $p=2$ and $q=1$ with $\alpha_{1}=\delta+1(\delta>-1), \alpha_{2}=1, \beta_{1}=1$, then

$$
\begin{aligned}
& W_{1}^{2}(\delta+1,1 ; 1) \phi(z) \\
= & D^{\delta} f(z)=\frac{z}{(1-z)^{\delta+1}} * \phi(z)
\end{aligned}
$$

is called Ruscheweyh derivative of order $\delta(\delta>-1)$.

From (8) now we define, Wright generalized hypergeometric harmonic function $f=h+\bar{g}$ of the form (1), as

$$
W_{q}^{p}\left[\alpha_{1}\right] f(z)=W_{q}^{p}\left[\alpha_{1}\right] h(z)+\overline{W_{q}^{p}\left[\alpha_{1}\right] g(z)}
$$

and we call this as Wright generalized operator on harmonic function.

Motivated by the earlier works of $[1,5,9-13]$ on the subject of harmonic functions, we introduce here a new subclass $W S_{H}\left(\left[\alpha_{1}\right], \gamma\right)$ of $H$.

For $0 \leq \gamma<1$, let $W S_{H}\left(\left[\alpha_{1}\right], \gamma\right)$ denote the subfamily of starlike harmonic functions $f \in H$ of the form (1) such that

$$
\frac{\partial}{\partial \theta}\left(\arg W_{q}^{p}\left[\alpha_{1}\right] f(z)\right)>\gamma
$$

equivalently

$$
\operatorname{Re}\left\{\frac{z\left(W_{q}^{p}\left[\alpha_{1}\right] h^{\prime}(z)\right)-\overline{z\left(W_{q}^{p}\left[\alpha_{1}\right] g^{\prime}(z)\right)}}{W_{q}^{p}\left[\alpha_{1}\right] h(z)+\overline{W_{q}^{p}\left[\alpha_{1}\right] g(z)}}\right\}>\gamma
$$

where $W_{q}^{p}\left[\alpha_{1}\right] f(z)$ is given by (11) and $z \in U$.

We also let $W V_{H}\left(\left[\alpha_{1}\right], \gamma\right)=W S_{H}\left(\left[\alpha_{1}\right], \gamma\right) \cap V_{H}$ where $V_{H}$ the class of harmonic functions with varying arguments introduced by Jahangiri and Silverman [10], consisting of functions $f$ of the form (1) in $H$ for which there exists a real number $\varphi$ such that

$$
\begin{aligned}
& \eta_{m}+(m-1) \varphi \\
\equiv & \pi(\bmod 2 \pi), \delta_{m}+(m-1) \varphi \\
\equiv & 0, m \geq 2,
\end{aligned}
$$

where $\eta_{m}=\arg \left(a_{m}\right)$ and $\delta_{m}=\arg \left(b_{m}\right)$.

In this paper we obtain a sufficient coefficient condition for functions $f$ given by (2) to be in the class $W S_{H}\left(\left[\alpha_{1}\right], \gamma\right)$. It is shown that this coefficient condition is necessary also for functions belonging to the class 
$W V_{H}\left(\left[\alpha_{1}\right], \gamma\right)$. Further, distortion results and extreme points for functions in $W V_{H}\left(\left[\alpha_{1}\right], \gamma\right)$ are also obtained.

\section{The Class $W S_{H}\left(\left[\alpha_{1}\right], \gamma\right)$}

We begin deriving a sufficient coefficient condition for the functions belonging to the class $W S_{H}\left(\left[\alpha_{1}\right], \gamma\right)$.

Theorem 1. Let $f=h+\bar{g}$ be given by (2). If

$$
\begin{aligned}
& \sum_{m=2}^{\infty}\left(\frac{m-\gamma}{1-\gamma}\left|a_{m}\right|+\frac{m+\gamma}{1-\gamma}\left|b_{m}\right|\right) . \\
& \sigma_{m}\left(\alpha_{1}\right) \leq 1-\frac{1+\gamma}{1-\gamma} b_{1}
\end{aligned}
$$

$0 \leq \gamma<1$, Then $f \in W S_{H}\left(\left[\alpha_{1}\right], \gamma\right)$.

Proof. We first show that if the inequality (15) holds for the coefficients of $f=h+\bar{g}$, then the required condition (13) is satisfied. Using (11) and (13), we can write

$$
\begin{aligned}
& \operatorname{Re}\left\{\frac{z\left(W_{q}^{p}\left[\alpha_{1}\right] h^{\prime}(z)\right)-\overline{z\left(W_{q}^{p}\left[\alpha_{1}\right] g^{\prime}(z)\right)}}{W_{q}^{p}\left[\alpha_{1}\right] h(z)+\overline{W_{q}^{p}\left[\alpha_{1}\right] g(z)}}\right\} \\
& =\operatorname{Re} \frac{A(z)}{B(z)}
\end{aligned}
$$

where

$$
A(z)=z\left(W_{q}^{p}\left[\alpha_{1}\right] h^{\prime}(z)\right)-\overline{z\left(W_{q}^{p}\left[\alpha_{1}\right] g^{\prime}(z)\right)}
$$

and

$$
B(z)=W_{q}^{p}\left[\alpha_{1}\right] h(z)+\overline{W_{q}^{p}\left[\alpha_{1}\right] g(z)}
$$

In view of the simple assertion that $\operatorname{Re}(w) \geq \gamma$ if and only if $|1-\gamma+w| \geq|1+\gamma-w|$, it is sufficient to show that

$$
|A(z)+(1-\gamma) B(z)|-|A(z)-(1+\gamma) B(z)| \geq 0 .
$$

Substituting for $A(z)$ and $B(z)$ the appropriate expressions in (16), we get

$$
|A(z)+(1-\gamma) B(z)|-|A(z)-(1+\gamma) B(z)|
$$

$$
\begin{gathered}
\geq(2-\gamma)|z|-\sum_{m=2}^{\infty}(m+1-\gamma) \sigma_{m}\left(\alpha_{1}\right)\left|a_{m}\right| \cdot \\
|z|^{m}-\sum_{m=1}^{\infty}(m-1+\gamma) \sigma_{m}\left(\alpha_{1}\right)\left|b_{m}\right||z|^{m} \\
-\gamma|z|-\sum_{m=2}^{\infty}(m-1-\gamma) \sigma_{m}\left(\alpha_{1}\right)\left|a_{m}\right| \cdot \\
|z|^{m}-\sum_{m=2}^{\infty}(m+1+\gamma) \sigma_{m}\left(\alpha_{1}\right)\left|b_{m}\right||z|^{m} \\
\geq 2(1-\gamma)|z|\left\{1-\sum_{m=2}^{\infty} \frac{m-\gamma}{1-\gamma} \sigma_{m}\left(\alpha_{1}\right)\left|a_{m}\right| .\right. \\
\left.|z|^{m-1}-\sum_{m=2}^{\infty} \frac{m+\gamma}{1-\gamma} \sigma_{m}\left(\alpha_{1}\right)\left|b_{m}\right||z|^{m-1}\right\} \\
\geq 2(1-\gamma)|z|\left\{1-\frac{1+\gamma}{1-\gamma} b_{1}-\right. \\
\left.\left(\sum_{m=2}^{\infty}\left[\frac{m-\gamma}{1-\gamma} \sigma_{m}\left(\alpha_{1}\right)\left|a_{m}\right|+\frac{m+\gamma}{1-\gamma} \sigma_{m}\left(\alpha_{1}\right)\left|b_{m}\right|\right]\right)\right\} \geq 0 .
\end{gathered}
$$

by virtue of the inequality (15). This implies that $f \in W S_{H}\left(\left[\alpha_{1}\right], \gamma\right)$.

Now we obtain the necessary and sufficient condition for function $f=h+\bar{g}$ be given with condition (14).

Theorem 2. Let $f=h+\bar{g}$ be given by (2) and for $0 \leq \gamma<1$, then $f \in W V_{H}\left(\left[\alpha_{1}\right], \gamma\right)$ if and only if

$$
\sum_{m=2}^{\infty}\left(\frac{m-\gamma}{1-\gamma}\left|a_{m}\right|+\frac{m+\gamma}{1-\gamma}\left|b_{m}\right|\right) \sigma_{m}\left(\alpha_{1}\right) \leq 1-\frac{1+\gamma}{1-\gamma} b_{1}
$$

Proof. Since $W V_{H}\left(\left[\alpha_{1}\right], \gamma\right) \subset W S_{H}\left(\left[\alpha_{1}\right], \gamma\right)$, we only need to prove the necessary part of the theorem. Assume that $f \in W V_{H}\left(\left[\alpha_{1}\right], \gamma\right)$, then by virture of (11) to (13), we obtain

$$
\operatorname{Re}\left\{\frac{z\left(W_{q}^{p}\left[\alpha_{1}\right] h^{\prime}(z)\right)-\overline{z\left(W_{q}^{p}\left[\alpha_{1}\right] g^{\prime}(z)\right)}}{W_{q}^{p}\left[\alpha_{1}\right] h(z)+\overline{W_{q}^{p}\left[\alpha_{1}\right] g(z)}}-\gamma\right\} \geq 0 .
$$

The above inequality is equivalent to

$$
\begin{gathered}
\operatorname{Re}\left\{\frac{z+\sum_{m=2}^{\infty}(m-\gamma) \sigma_{m}\left(\alpha_{1}\right)\left|a_{m}\right| z^{m}-\sum_{m=1}^{\infty}(m+\gamma) \sigma_{m}\left(\alpha_{1}\right)\left|b_{m}\right| \bar{z}^{m}}{z+\sum_{m=2}^{\infty} \sigma_{m}\left(\alpha_{1}\right)\left|a_{m}\right| z^{m}+\sum_{m=1}^{\infty} \sigma_{m}\left(\alpha_{1}\right)\left|b_{m}\right| \bar{z}^{m}}\right\} \\
=\operatorname{Re}\left\{\frac{(1-\gamma)+\sum_{m=2}^{\infty}(m-\gamma) \sigma_{m}\left(\alpha_{1}\right)\left|a_{m}\right| z^{m-1}-\frac{\bar{z}}{z} \sum_{m=1}^{\infty}(m+\gamma) \sigma_{m}\left(\alpha_{1}\right)\left|b_{m}\right| \bar{z}^{m-1}}{1+\sum_{m=2}^{\infty} \sigma_{m}\left(\alpha_{1}\right)\left|a_{m}\right| z^{m-1}+\frac{\bar{z}}{z} \sum_{m=1}^{\infty} \sigma_{m}\left(\alpha_{1}\right)\left|b_{m}\right| \bar{z}^{m-1}}\right\} \geq 0 .
\end{gathered}
$$


This condition must hold for all values of $z$, such that $|z|=r<1$. Upon choosing $\varphi$ according to (14) we must have (18).

If (17) does not hold, then the numerator in (18) is negative for $r$ sufficiently close to 1 . Therefore, there exists a point $z_{o}=r_{o}$ in $(0,1)$ for which the quotient in (18) is negative. This contradicts our assumption that $f \in W V_{H}\left(\left[\alpha_{1}\right], \gamma\right)$. We thus conclude that it is both necessary and sufficient that the coefficient bound inequality (17) holds true when $f \in W V_{H}\left(\left[\alpha_{1}\right], \gamma\right)$. This completes the proof of Theorem 2 .

If we put $\varphi=\frac{2 \pi}{k}$ in (14), then Theorem 2 gives the following corollary.

Corollary 1. A necessary and sufficient condition for $f=h+\bar{g}$ satisfying (17) to be starlike is that $\arg \left(a_{m}\right)=$ $\pi-2(m-1) \pi / k$, and $\arg \left(b_{m}\right)=2 \pi-2(m-1) \pi / k,(k=1$, $2,3, \ldots)$.

\section{Distortion Bounds and Extreme Points}

In this section we obtain the distortion bounds for the functions $f \in W V_{H}\left(\left[\alpha_{1}\right], \gamma\right)$ that lead to a covering result for the family $W V_{H}\left(\left[\alpha_{1}\right], \gamma\right)$.

Theorem 3. If $f \in W V_{H}\left(\left[\alpha_{1}\right], \gamma\right)$ then

$$
f(z) \leq\left(1+\left|b_{1}\right|\right) r+\frac{1}{\sigma_{2}\left(\alpha_{1}\right)}\left(\frac{1-\gamma}{2-\gamma}-\frac{1+\gamma}{2-\gamma}\left|b_{1}\right|\right) r^{2}
$$

and

$$
f(z) \geq\left(1-\left|b_{1}\right|\right) r-\frac{1}{\sigma_{2}\left(\alpha_{1}\right)}\left(\frac{1-\gamma}{2-\gamma}-\frac{1+\gamma}{2-\gamma}\left|b_{1}\right|\right) r^{2} .
$$

Proof. We will only prove the right-hand inequality of the above theorem. The arguments for the left-hand inequality are similar and so we omit it. Let $f \in W V_{H}$. $\left(\left[\alpha_{1}\right], \gamma\right)$ taking the absolute value of $f$, we obtain

$$
\begin{aligned}
& |f(z)| \\
& \leq\left(1+\left|b_{1}\right|\right) r+\sum_{m=2}^{\infty}\left(\left|a_{m}\right|+\left|b_{m}\right|\right) r^{m}
\end{aligned}
$$

$$
\leq\left(1+\left|b_{1}\right|\right) r+r^{2} \sum_{m=2}^{\infty}\left(\left|a_{m}\right|+\left|b_{m}\right|\right) .
$$

This implies that

$$
\begin{aligned}
& |f(z)| \leq\left(1+\left|b_{1}\right|\right) r+\frac{1}{\sigma_{2}\left(\alpha_{1}\right)}\left(\frac{1-\gamma}{2-\gamma}\right) . \\
& \sum_{m=2}^{\infty}\left(\left(\frac{2-\gamma}{1-\gamma}\right) \sigma_{2}\left(\alpha_{1}\right)\left|a_{m}\right|+\left(\frac{2-\gamma}{1-\gamma}\right) \sigma_{2}\left(\alpha_{1}\right)\left|b_{m}\right|\right) r^{2} \\
& \quad \leq\left(1+\left|b_{1}\right|\right) r+\frac{1}{\sigma_{2}\left(\alpha_{1}\right)}\left(\frac{1-\gamma}{2-\gamma}\right)\left[1-\frac{1+\gamma}{1-\gamma}\left|b_{1}\right|\right] r^{2} \\
& \quad \leq\left(1+\left|b_{1}\right|\right) r+\frac{1}{\sigma_{2}\left(\alpha_{1}\right)}\left(\frac{1-\gamma}{2-\gamma}-\frac{1+\gamma}{2-\gamma}\left|b_{1}\right|\right) r^{2},
\end{aligned}
$$

which establish the desired inequality.

As consequences of the above theorem and corollary 1, we state the following corollary.

Corollary 2. Let $f=h+\bar{g}$ and of the form (2) be so that $f \in W V_{H}\left(\left[\alpha_{1}\right], \gamma\right)$. Then

$$
\begin{aligned}
& \left\{w:|w|<\frac{2 \sigma_{2}\left(\alpha_{1}\right)-1-\left[\sigma_{2}\left(\alpha_{1}\right)-1\right] \gamma}{(2-\gamma) \sigma_{2}\left(\alpha_{1}\right)}\right. \\
& \left.-\frac{2 \sigma_{2}\left(\alpha_{1}\right)-1-\left[\sigma_{2}\left(\alpha_{1}\right)+1\right] \gamma}{(2-\gamma) \sigma_{2}\left(\alpha_{1}\right)} b_{1}\right\} \subset f(U) .
\end{aligned}
$$

For a compact family, the maximum or minimum of the real part of any continuous linear functional occurs at one of the extreme points of the closed convex hull. Unlike many other classes, characterized by necessary and sufficient coefficient conditions, the family $W V_{H}\left(\left[\alpha_{1}\right]\right.$, $\gamma)$ is not a convex family. Nevertheless, we may still apply the coefficient characterization of the $W V_{H}\left(\left[\alpha_{1}\right]\right.$, $\gamma$ ) to determine the extreme points.

Theorem 4. The closed convex hull of $W V_{H}\left(\left[\alpha_{1}\right], \gamma\right)$ (denoted by clco $\left.W V_{H}\left(\left[\alpha_{1}\right], \gamma\right)\right)$ is

$$
\begin{aligned}
& \left\{f(z)=z+\sum_{m=2}^{\infty}\left|a_{m}\right| z^{m}+\overline{\sum_{m=1}^{\infty}\left|b_{m}\right| z^{m},}\right. \\
& \left.: \sum_{m=2}^{\infty} m\left[\left|a_{m}\right|+\left|b_{m}\right|\right]<1-b_{1}\right\} .
\end{aligned}
$$

$$
\frac{(1-\gamma)-(1+\gamma) b_{1}-\left(\sum_{m=2}^{\infty}(m-\gamma) \sigma_{m}\left(\alpha_{1}\right)\left|a_{m}\right| r^{m-1}+(m+\gamma) \sigma_{m}\left(\alpha_{1}\right)\left|b_{m}\right| r^{m-1}\right)}{1+\left|b_{1}\right|+\left(\sum_{m=2}^{\infty} \sigma_{m}\left(\alpha_{1}\right)\left|a_{m}\right|+\sum_{m=1}^{\infty} \sigma_{m}\left(\alpha_{1}\right)\left|b_{m}\right|\right) r^{m-1}} \geq 0 .
$$


By setting $\lambda_{m}=\frac{1-\gamma}{(m-\gamma) \sigma_{m}\left(\alpha_{1}\right)}$ and $\mu_{m}=\frac{1-\gamma}{(m+\gamma) \sigma_{m}\left(\alpha_{1}\right)}$, then for $b_{1}$ fixed, the extreme points for $\operatorname{clco} W V_{H}\left(\left[\alpha_{1}\right]\right.$, $\gamma)$ are

$$
\left\{z+\lambda_{m} x z^{m}+\overline{b_{1} z}\right\} \cup\left\{z+\overline{b_{1} z+\mu_{m} x z^{m}}\right\}
$$

where $m \geq 2$ and $|x|=1-\left|b_{1}\right|$.

Proof. Any function $f$ in clco $W V_{H}\left(\left[\alpha_{1}\right], \gamma\right)$ be expressed as

$$
f(z)=z+\sum_{m=2}^{\infty}\left|a_{m}\right| e^{i \eta_{m}} z^{m}+\overline{b_{1} z}+\overline{\sum_{m=2}^{\infty}\left|b_{m}\right| e^{i \delta_{m}} z^{m}},
$$

where the coefficient satisfy the inequality (15). Set $h_{1}(z)=z, \quad g_{1}(z)=b_{1} z, \quad h_{m}(z)=z+\lambda_{m} e^{i \eta_{m}} z^{m}, \quad g_{m}(z)=b_{1} z$ $+\mu_{m} e^{i \mu_{m}} z^{m}$, for $m=2,3, \ldots$ Writing

$$
X_{m}=\frac{\left|a_{m}\right|}{\lambda_{m}}, Y_{m}=\frac{\left|b_{m}\right|}{\mu_{m}} m=2,3 \ldots \text { and } X_{1}=1-\sum_{m=2}^{\infty} X_{m} ;
$$

$Y_{1}=1-\sum_{m=2}^{\infty} Y_{m} ; \quad$ we get

$$
f(z)=\sum_{m=1}^{\infty}\left[X_{m} h_{m}(z)+Y_{m} g_{m}(z)\right] .
$$

In particular, putting

$$
f_{1}(z)=z+\overline{b_{1} z}
$$

and

$$
\begin{gathered}
f_{m}(z)=z+\lambda_{m} x z^{m}+\overline{b_{1} z}+\overline{\mu_{m} y z^{m}}, \\
\left(m \geq 2,|x|+|y|=1-\left|b_{1}\right|\right)
\end{gathered}
$$

We see that extreme points of functions in $c l c o W V_{H}$. $\left(\left[\alpha_{1}\right], \gamma\right) \subset\left\{f_{m}(z)\right\}$. To see that $f_{m}$ is not an extreme point if both $|x| \neq 0$ and $|y| \neq 0$, we will show that it can then also be expressed as a convex linear combinations of functions in $\operatorname{clco} W V_{H}\left(\left[\alpha_{1}\right], \gamma\right)$. Without loss of generality, assume $|x| \geq|y|$. Choose $\in>0$ small enough so that $\in<\frac{|x|}{|y|}$. Set $A=1+\epsilon$ and $B=1-\left|\frac{\in x}{y}\right|$. We then see that both

$$
t_{1}(z)=z+\lambda_{m} A x z^{m}+\overline{b_{1} z+\mu_{m} B y z^{m}}
$$

and

$$
t_{2}(z)=z+\lambda_{m}(2-A) x z^{m}+\overline{b_{1} z+\mu_{m}(2-B) y z^{m}}
$$

are in clco $W V_{H}\left(\left[\alpha_{1}\right], \gamma\right)$, and that $f_{m}(z)=\frac{1}{2}\left\{t_{1}(z)\right.$ $\left.+t_{2}(z)\right\}$. The extremal coefficient bounds show that functions of the form (19) are the extreme points for clco $W V_{H}\left(\left[\alpha_{1}\right], \gamma\right)$, and so the proof is complete.

\section{Inclusion Relation}

Following Avici and Zlotkiewicz [9] (see also Ruscheweyh [14]), we refer to the $\delta$-neighborhood of the function $f(z)$ defined by (2) to be the set of functions $F$ for which

$$
\begin{aligned}
N_{\delta}(f):= & \left\{F(z)=z+\sum_{m=2}^{\infty} A_{m} z^{m}+\sum_{m=2}^{\infty} \overline{B_{m} z^{m}},\right. \\
& \left.\sum_{m=2}^{\infty} m\left(\left|a_{m}-A_{m}\right|+\left|b_{m}-B_{m}\right|\right)+\left|b_{1}-B_{1}\right| \leq \delta\right\} .
\end{aligned}
$$

In our case, let us define the generalized $\delta$-neighborhood of $\mathrm{f}$ to be the set

$$
\begin{aligned}
& N_{\delta}(f):=\left\{F: \sum_{m=2}^{\infty} \sigma_{m}\left(\alpha_{1}\right)\left[(m-\gamma)\left|a_{m}-A_{m}\right|\right.\right. \\
& \left.\left.+(m+\gamma)\left|b_{m}-B_{m}\right|\right]+(1-\gamma)\left|b_{1}-B_{1}\right| \leq(1-\gamma) \delta\right\} .
\end{aligned}
$$

Theorem 5. Let $f$ be given by (2). If $f$ satisfies the conditions

$$
\begin{aligned}
& \sum_{m=2}^{\infty} m(m-\gamma)\left|a_{m}\right| \sigma_{m}\left(\alpha_{1}\right) \\
+ & \sum_{m=1}^{\infty} m(m+\gamma)\left|b_{m}\right| \sigma_{m}\left(\alpha_{1}\right) \leq 1-\gamma,
\end{aligned}
$$

$0 \leq \gamma<1$ and $0 \leq \gamma<1$, then $N_{\delta}(f) \subset W S_{H}\left(\left[\alpha_{1}\right], \gamma\right)$.

Proof. Let $f$ satisfy (22) and $F(z)$ be given by

$$
F(z)=z+\sum_{m=2}^{\infty} A_{m} z^{m}+\sum_{m=2}^{\infty} \overline{B_{m} z^{m}},
$$

which belongs to $N(f)$. We obtain

$$
\begin{aligned}
(1+\gamma) & \left|B_{1}\right|+\sum_{m=2}^{\infty}(m-\gamma)\left|A_{m}\right|+(m-\gamma)\left|B_{m}\right| \sigma_{m}\left(\alpha_{1}\right) \\
\leq & (1+\gamma)\left|B_{1}-b_{1}\right|+(1+\gamma)\left|b_{1}\right| \\
& +\sum_{m=2}^{\infty} \sigma_{m}\left(\alpha_{1}\right)(m-\gamma)\left|A_{m}-a_{m}\right| \\
& +(m+\gamma)\left|B_{m}-b_{m}\right| \\
& +\sum_{m=2}^{\infty} \sigma_{m}\left(\alpha_{1}\right)\left[(m-\gamma)\left|a_{m}\right|+(m+\gamma)\left|b_{m}\right|\right] \\
\leq & (1-\gamma) \delta+(1+\gamma)\left|b_{1}\right| \\
+ & \frac{1}{2-\gamma} \sum_{m=2}^{\infty} m \sigma_{m}\left(\alpha_{1}\right)\left((m-\gamma)\left|a_{m}\right|+(m+\gamma)\left|b_{m}\right|\right)
\end{aligned}
$$




$$
\leq(1-\gamma) \delta+(1+\gamma)\left|b_{1}\right|+\frac{1}{2-\gamma}\left((1-\gamma)-(1+\gamma)\left|b_{1}\right|\right) \leq 1-\gamma .
$$

Hence for $\delta=\frac{1-\gamma}{2-\gamma}\left(1-\frac{1+\gamma}{1-\gamma}\left|b_{1}\right|\right)$, we infer that $W S_{H}\left(\left[\alpha_{1}\right], \gamma\right)$ which concludes the proof of Theorem 5.

Now, we will examine the closure properties of the class $W V_{H}\left(\left[\alpha_{1}\right], \gamma\right)$ under the generalized Bernardi-Libera-Livingston integral operator $L_{c}(f)$ which is defined by

$$
L_{c}(f)=\frac{c+1}{z^{c}} \int_{0}^{z} t^{c-1} f(t) d t, c>-1 .
$$

Theorem 6. Let $f \in W V_{H}\left(\left[\alpha_{1}\right], \gamma\right)$. Then $L_{c}(f) \in$ $W V_{H}\left(\left[\alpha_{1}\right], \gamma\right)$.

Proof. From the representation of $L_{c}(f)$, it follows that

$$
\begin{aligned}
L_{c}(f)= & \frac{c+1}{z^{c}} \int_{0}^{z} t^{c-1}[h(t)+\overline{g(t)}] d t \\
= & \frac{c+1}{z^{c}}\left(\int_{0}^{z} t^{c-1}\left(t-\sum_{m=2}^{\infty} a_{m} t^{m}\right) d t\right. \\
& \left.+\int_{0}^{z} t^{c-1}\left(\sum_{m=1}^{\infty} b_{m} t^{m}\right) d t\right) \\
= & z-\sum_{m=2}^{\infty} A_{m} z^{m}+\sum_{m=1}^{\infty} B_{m} z^{m},
\end{aligned}
$$

where

$$
A_{m}=\frac{c+1}{c+m} a_{m} ; \quad B_{m}=\frac{c+1}{c+m} b_{m} .
$$

Therefore

$$
\begin{aligned}
& \sum_{m=1}^{\infty}\left(\frac{m-\gamma}{1-\gamma} \frac{c+1}{c+m}\left|a_{m}\right|+\frac{m+\gamma}{1-\gamma} \frac{c+1}{c+m}\left|b_{m}\right|\right) \sigma_{m}\left(\alpha_{1}\right) \\
\leq & \sum_{m=1}^{\infty}\left(\frac{m-\gamma}{1-\gamma}\left|a_{m}\right|+\frac{m+\gamma}{1-\gamma}\left|b_{m}\right|\right) \sigma_{m}\left(\alpha_{1}\right) \leq 2(1-\gamma) .
\end{aligned}
$$

Since $f \in W V_{H}\left(\left[\alpha_{1}\right], \gamma\right)$, therefore by Theorem 2, $L_{c}(f) \in W V_{H}\left(\left[\alpha_{1}\right], \gamma\right)$.

Theorem 7. For $0 \leq \delta \leq \gamma<1$, let $f \in W V_{H}\left(\left[\alpha_{1}\right], \gamma\right)$ and $F \in W V_{H}\left(\left[\alpha_{1}\right], \delta\right)$. Then $\left(f^{*} F\right) \in W V_{H}\left(\left[\alpha_{1}\right], \gamma\right) \subset$ $W V_{H}\left(\left[\alpha_{1}\right], \delta\right)$.

Proof. Let

$$
f(z)=z-\sum_{m=2}^{\infty} a_{m} z^{m}+\sum_{m=1}^{\infty} \bar{b}_{m} \bar{z}^{m} \in W V_{H}\left(\left[\alpha_{1}\right], \gamma\right)
$$

and

$$
F(z)=z-\sum_{m=2}^{\infty} A_{m} z^{m}+\sum_{m=1}^{\infty} \bar{B}_{m} \bar{z}^{m} \in W V_{H}\left(\left[\alpha_{1}\right], \delta\right)
$$

Then

$$
f(z)^{*} F(z)=z-\sum_{m=2}^{\infty} A_{m} a_{m} z^{m}+\sum_{m=1}^{\infty} \bar{B}_{m} \bar{b}_{m} \bar{z}^{m}
$$

For $\left(f^{*} F\right) \in W V_{H}\left(\left[\alpha_{1}\right], \delta\right)$ we note that $\left|A_{m}\right| \leq 1$ and $\left|B_{m}\right| \leq 1$. Now by Theorem 2, we have

$$
\begin{gathered}
\sum_{m=2}^{\infty} \frac{(m-\delta) \sigma_{m}\left(\alpha_{1}\right)}{1-\delta}\left|a_{m}\right|\left|A_{m}\right| \\
+\sum_{m=1}^{\infty} \frac{(m+\delta) \sigma_{m}\left(\alpha_{1}\right)}{1-\delta}\left|b_{m}\right|\left|B_{m}\right| \\
\leq \sum_{m=2}^{\infty} \frac{(m-\delta) \sigma_{m}\left(\alpha_{1}\right)}{1-\delta}\left|a_{m}\right|+\sum_{m=1}^{\infty} \frac{(m+\delta) \sigma_{m}\left(\alpha_{1}\right)}{1-\delta}\left|b_{m}\right| \\
\leq \sum_{m=2}^{\infty} \frac{(m-\gamma) \sigma_{m}\left(\alpha_{1}\right)}{1-\gamma}\left|a_{m}\right|+\sum_{m=1}^{\infty} \frac{(m+\gamma) \sigma_{m}\left(\alpha_{1}\right)}{1-\gamma}\left|b_{m}\right| \leq 1 .
\end{gathered}
$$

Therefore $\left(f^{*} F\right) \in W V_{H}\left(\left[\alpha_{1}\right], \gamma\right) \subset W V_{H}\left(\left[\alpha_{1}\right], \delta\right)$ and since the above inequality bounded by $2(1-\gamma)$ while $2(1-\gamma) \leq 2(1-\delta)$.

\section{Concluding Remarks}

The various results presented in this paper would provide interesting extensions and generalizations of those considered earlier for simpler harmonic function classes (see $[10,12,13])$. The details involved in the derivations of such specializations of the results presented in this paper are fairly straight-forward.

\section{References}

[1] J. Clunie and T. Sheil-Small, "Harmonic Univalent Functions," Annales Academiae Scientiarum Fennicae, Series A I, Mathematica, Vol. 9, 1984, pp. 3-25.

[2] E. M. Wright, "The Asymptotic Expansion of the Generalized Hypergeometric Function," Proceedings of the London Mathematical Society, Vol. 46, 1946, pp. 389-408.

[3] J. Dziok and H. M. Srivastava, "Certain Subclasses of Analytic Functions Associated with the Generalized Hypergeometric Function," Integral Transforms and Special Functions, Vol. 14, No. 1, 2003, pp. 7-18.

[4] J. Dziok and R. K. Raina, "Families of Analytic Functions Associated with the Wright Generalized Hypergeometric Function," Demonstratio Mathematica, Vol. 37, No. 3, 2004, pp. 533-542.

[5] J. M. Jahangiri, "Harmonic Functions Starlike in the Unit Disc," Journal of Mathematical Analysis and Applications, Vol. 235, No. 2, 1999, pp. 470-477. 
[6] B. C. Carlson and D. B. Shaffer, "Starlike and Prestarlike Hypergeometric Functions," SIAM Journal on Mathematical Analysis, Vol. 15, No. 4, 1984, pp. 737-745.

[7] S. Ruscheweyh, "New Criteria for Univalent Functions," Proceedings of the American Mathematical Society, Vol. 49, No. 1, 1975, pp. 109-115.

[8] H. M. Srivastava and S. Owa, "Some Characterization and Distortion Theorems Involving Fractional Calculus, Generalized Hypergeometric Functions, Hadamard Products, Linear Operators and Certain Subclasses of Analytic Functions," Nagoya Mathematics Journal, Vol. 106, 1987, pp. 1-28.

[9] Y. Avici and E. Zlotkiewicz, "On Harmonic Univalent Mappings," Annales Universitatis Mariae Curie-Skłodowska, Sectio A, Vol. 44, 1990, pp. 1-7.

[10] J. M. Jahangiri and H. Silverman, "Harmonic Univalent Functions with Varying Arguments," International Jour- nal of Applied Mathematics, Vol. 8, No. 3, 2002, pp. 267 275.

[11] J. M. Jahangiri, G. Murugusundaramoorthy and K. Vijaya, "Starlikeness of Rucheweyh Type Harmonic Univalent Functions," Journal of the Indian Academy of Mathematics, Vol. 26, No. 1, 2004, pp. 191-200.

[12] G. Murugusundaramoorthy, "A Class of Ruscheweyh-Type Harmonic Univalent Functions with Varying Arguments," Southwest Journal of Pure and Applied Mathematics, No. 2, 2003, pp. 90-95.

[13] K. Vijaya, "Studies on Certain Subclasses of Harmonic Functions," Ph.D. Thesis, Vellore Institute of Technology University, Vellore, September 2006.

[14] S. Ruscheweyh, "Neighborhoods of Univalent Functions," Proceedings of the American Mathematical Society, Vol. 81, No. 4, 1981, pp. 521-528. 